\title{
Design and Optimization of Annular Flow Electromagnetic Measurement System for Drilling Engineering
}

\author{
Liang Ge $\mathbb{D}^{1},{ }^{1,2,3}$ Hailong Li, ${ }^{2}$ Qing Wang, ${ }^{3}$ Guohui Wei, ${ }^{2} \mathrm{Ze} \mathrm{Hu},{ }^{2}$ Junbi Liao, ${ }^{1}$ and Junlan $\mathrm{Li}^{4}$ \\ ${ }^{1}$ School of Manufacturing Science and Engineering, Sichuan University, Chengdu 610065, China \\ ${ }^{2}$ College of Mechanical and Electronic Engineering, Southwest Petroleum University, Chengdu 610500, China \\ ${ }^{3}$ Department of Engineering, Durham University, Durham DH1 3LE, UK \\ ${ }^{4}$ Southwest Branch, Engineering Design Co., CNPC, Chengdu 610413, China \\ Correspondence should be addressed to Liang Ge; cgroad@163.com
}

Received 28 June 2017; Accepted 18 October 2017; Published 25 February 2018

Academic Editor: Bruno Andò

Copyright ( 2018 Liang Ge et al. This is an open access article distributed under the Creative Commons Attribution License, which permits unrestricted use, distribution, and reproduction in any medium, provided the original work is properly cited.

\begin{abstract}
Using the downhole annular flow measurement system to get real-time information of downhole annular flow is the core and foundation of downhole microflux control drilling technology. The research work of electromagnetic flowmeter in recent years creates a challenge to the design of downhole annular flow measurement. This paper proposes a design and optimization of annular flow electromagnetic measurement system for drilling engineering based on the finite element method. Firstly, the annular flow measuring and optimization principle are described. Secondly, a simulation model of an annular flow electromagnetic measurement system with two pairs of coil is built based on the fundamental equation of electromagnetic flowmeter by COMSOL. Thirdly, simulations of the structure of excitation system of the measurement system are carried out, and simulations of the size of the electrode's radius are also carried out based on the optimized structure, and then all the simulation results are analyzed to evaluate the optimization effect based on the evaluation indexes. The simulation results show that optimized shapes of the excitation system and electrode size can yield a better performance in the annular flow measurement.
\end{abstract}

\section{Introduction}

In recent decades, oil and gas exploration is being carried out in some extremely harsh and challenging environmental conditions [1]. Drilling safety issues become increasingly prominent when exploring complex and deep formations. Downhole microflux control drilling technology can effectively solve drilling accident such as kick and lose in narrow density window drilling scenarios, so using the measurement system to get the real-time information of downhole annular flow is the core and foundation of downhole microflux control drilling technology.

A huge array of flow technology options is on offer which provides options in selecting the correct annular flow measurement for the application of drilling engineering. A broad range of factors regarding the special environment of downhole drilling, such as downhole space, velocity profile, temperature, and fluid properties, should be considered. Electromagnetic measurement has the advantages of simple structure, no moving parts, and no obstruction of fluid flow throttle parts. Also, the flow path does not cause any additional pressure loss, and it does not cause wear or blockage, in particular when measuring slurry with solid particles, sewage and other liquid-solid two-phase bodies, or a variety of viscous slurry, and so on. In addition, because the structure has no moving parts, so any corrosion will be attached to the insulation lining. After selecting corrosion-resistant electrode material with a very good corrosion resistance, it can be used for a variety of corrosive media measurements. In 2017, Liang et al. propose a new method for an annular flow measurement system based on the electromagnetic induction principle [2]. However, this paper does not describe how to design and optimize the downhole annular flow electromagnetic measurement system. Based on the above reasons, this 
study mainly focuses on the design and optimization of downhole annular flow electromagnetic measurement system for drilling engineering.

For the traditional electromagnetic flowmeter used in a round pipe, the signal voltage is dependent on the average flow velocity, the magnetic flux density, and the pipe diameter. The signal voltage is expected to be linearly related to the average flow velocity if the magnetic field is a uniform magnetic field. In this ideal case, the flow rate can be considered to be immune to the velocity profile of the pipe flow, especially when the flow has been fully developed. However, it is difficult for the annular flow electromagnetic measurement system to yield a uniform magnetic field for an annular flow path, and also, the velocity profile of the annular flow also cannot be considered axisymmetrically distributed under this special drilling environment. These effects affect the accuracy of the annular flow electromagnetic measurement system. According to Bevie's vector weight function theory [3], if the result of the magnetic flux density cross-product density of virtual current is constant, the annular flow electromagnetic measurement system can be considered to be immune to the velocity profile of the annular flow. When the electrode and the structure of the flow path are fixed, the density of the virtual current is fixed. So the shape of the excitation system can be derived based on this constant condition. Similarly, when the shape of excitation system and the structure of flow path are fixed, the density of virtual current only depends on the electrodes. In this case, the size of the electrode was selected to be optimized to reduce the distortion caused by the velocity profile.

In the past few decades, great efforts about the structure of excitation systems and electrodes have been made to reduce the effects of the velocity profile. Horner B improved the measurement accuracy of flow measurement by increasing the number of electrodes [4, 5]. Michalski et al. investigated the design of the coils of an electromagnetic flowmeter in 1998 [6]. Wang et al. analyzed the relationship between velocity profile and distribution of induced potential for an electromagnetic flow meter in 2007 [7]. An optimum excitation coil for an open channel electromagnetic flowmeter was reported by Michalski et al. in 2001 [8]. Vieira et al. developed an enhanced ellipsoid method for electromagnetic device optimization and design in 2010 [9]. At the same time, some simulation methods were designed with the development of finite element software for multifields. Lim and Choong analyzed the relative errors in evaluating the electromagnetic flowmeter signal using the weight function method and the finite volume method [10]. Michalski et al. applied $3 \mathrm{D}$ approach to designing the excitation coil of an electromagnetic flowmeter in 2012 [11]. Yin et al. investigated the theoretical and numerical approaches to the sensitivity calculation of a novel contactless inductive flow tomography [12]. Although great efforts about the structure of the excitation system and electrodes have been made to reduce the effects of velocity profile, most of the design and optimization was focused on the round pipe. The main objective of this paper is to design and optimize the downhole annular flow electromagnetic measurement system with two pairs of electrodes using the method of numerical finite element analysis.

\section{Background Theory of the Annular Four- Electrode Electromagnetic Flowmeter}

Following Faraday's law, the flow of a conductive liquid through a magnetic field will cause a voltage signal to be sensed by electrodes located on the flow pipe walls. Faraday's formula can be expressed as

$$
E=B D \bar{v}
$$

where $E$ is the signal voltage in a conductor, $\bar{v}$ is the average flow velocity, $B$ is the magnetic flux density, and $D$ is the pipe diameter.

Equation (1) indicates that the signal voltage is dependent on the average flow velocity, the magnetic flux density, and the pipe diameter.

The signal voltage is expected to be linearly related to the average flow velocity if the magnetic field is a uniform magnetic field. So (1) can be rewritten as

$$
E=C \bar{v} .
$$

Here, $C$ represents a constant.

However, the annular flow electromagnetic measurement system is difficult to yield a uniform magnetic field for an annular flow path, and the velocity profile of the annular flow also cannot be considered axisymmetrically distributed under this special drilling environment. This affects the accuracy of the annular flow electromagnetic measurement system. So the design and optimization of the downhole annular flow electromagnetic measurement system with two pairs of electrodes cannot be investigated based on the traditional Faraday theory.

According to Bevir's theory [3], the theoretical expression of the induced potential in annular flow electromagnetic measurement system can be given as a volume integral of the weight function vector $\mathbf{W}$ and the annular flow velocity $\vec{v}$ as follows:

$$
U=\int_{\tau} \mathbf{W} \cdot \vec{v} \mathbf{d} \tau,
$$

where $\mathbf{W}$ is given by $\mathbf{W}=\overrightarrow{\mathbf{B}} \times \overrightarrow{\mathbf{j}}$.

Here, $U$ is the induced potential, $\mathbf{W}$ is the weight function vector, $\vec{v}$ is the velocity of the annular flow, $\overrightarrow{\mathbf{B}}$ is the magnetic flux vector, $\overrightarrow{\mathbf{j}}$ is the virtual current vector, and $\tau$ is the integration of annular volume. The velocity of the annular flow weight function vector $\mathbf{W}$ is dependent on the magnetic flux density vector $\overrightarrow{\mathbf{B}}$ and virtual current vector $\overrightarrow{\mathbf{j}}$.

According to Bevie's vector weight function theory, if the result of the magnetic flux density cross-product density of virtual current is constant, the annular flow electromagnetic measurement system can be considered to be immune to the velocity profile of the annular flow. When the electrode and the structure of the flow path are fixed, the density of virtual current is fixed. Thus, the shape of excitation system can be derived based on this constant condition. Similarly, when the shape of the excitation system and the structure of the 


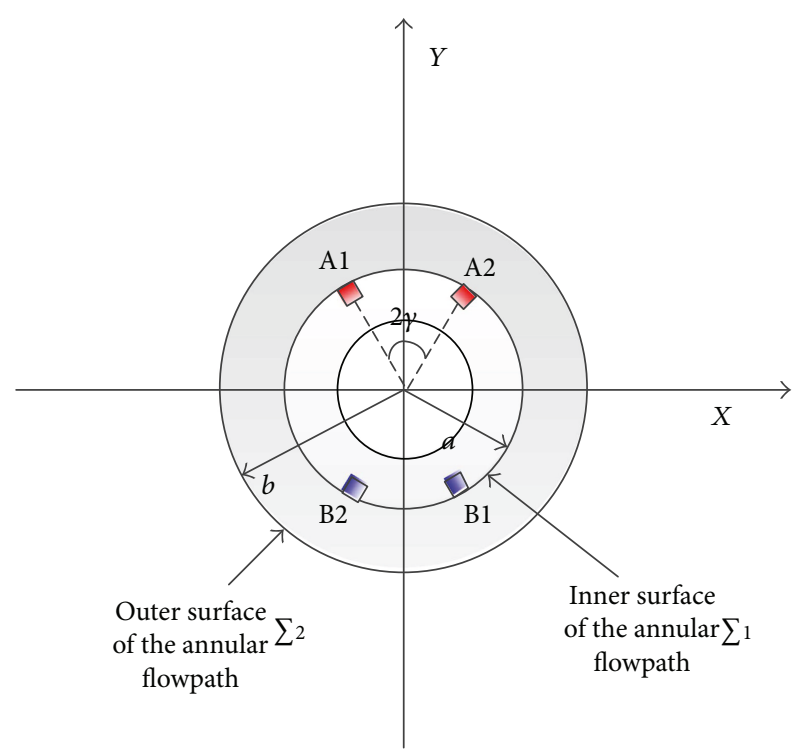

FIGURE 1: The schematic diagram of the annular four-electrode flow electromagnetic measurement system and the flow path.

flow path are fixed, the density of the virtual current only depends on the electrodes. In this case, the radius of the electrode was selected to be optimized to reduce the distortion caused by the velocity profile.

Figure 1 shows a schematic diagram of the downhole annular four-electrode electromagnetic flowmeter and the flow path. A1, A2, B1, and B2 represent four electrodes placed on the outer wall at intervals of $2 \gamma$ inside the annular flow path ( $\gamma=45^{\circ}$ while in simulation), $a$ and $b$ are the distance of the inner and outer surfaces to the center, and $\sum 1$ and $\sum 2$ represent the inner and outer surfaces of the annular flow path.

To simplify the boundary conditions, we suppose that the electronic conductivity of the outer surface of the annular flow path is much smaller than the flow. The partial differential equation and the boundary conditions of the outer surface of the annular flow path and electrodes can be written as follows:

$$
\begin{aligned}
\frac{\partial^{2} G}{\partial r^{2}}+\frac{1}{r} \frac{\partial G}{\partial r}+\frac{1}{r^{2}} \frac{\partial^{2} G}{\partial \phi^{2}} & =0 \\
\left.\frac{\partial G}{\partial r}\right|_{r=b} & =0
\end{aligned}
$$

By using the segregation variable method, (4) can be expressed as

$$
\begin{aligned}
r^{2} \frac{d^{2} R}{d r^{2}}+r \frac{d R}{d r}-K R & =0 \\
\frac{d^{2} \Phi}{d \phi^{2}}+K \phi & =0
\end{aligned}
$$

When $K=n^{2}$, by solving differential equations, the results can be written as

$$
\begin{aligned}
& R=C_{0}+D_{0} \ln r+\sum_{n=1}^{\infty}\left(C_{n} r^{n}+D_{n} r^{-n}\right), \\
& \Phi=\sum_{n=1}^{\infty}\left(A_{n} \sin n \phi+B_{n} \cos n \phi\right) .
\end{aligned}
$$

Equation (6) can be transformed into the following equation:

$$
\begin{aligned}
G= & C_{0}+D_{0} \ln r \\
& +\sum_{n=1}^{\infty}\left(C_{n} r^{n}+D_{n} r^{-n}\right)\left(A_{n} \sin n \phi+B_{n} \cos n \phi\right), \\
\frac{\partial G}{\partial r}= & D_{0} \frac{1}{r} \\
& +\sum_{n=1}^{\infty} n r^{-n-1}\left(C_{n} r^{2 n}-D_{n}\right)\left(A_{n} \sin n \phi+B_{n} \cos n \phi\right) .
\end{aligned}
$$

If $\mathrm{A} 1$ and $\mathrm{B} 1$ are the positive electrodes and $\mathrm{A} 2$ and $\mathrm{B} 2$ are the negative electrodes, the boundary conditions of the inner surface of the annular flow path and electrodes can be written as follows:

$$
\left.\frac{\partial G}{\partial r}\right|_{r=a}=\left\{\begin{array}{l}
\frac{\delta(\phi-(\pi / 2)+\gamma)}{(2 a)}, \\
\frac{-\delta(\phi-(\pi / 2)-\gamma)}{(2 a)}, \\
\frac{-\delta(\phi+(\pi / 2)+\gamma)}{(2 a)}, \\
(2 a)
\end{array}\right.
$$

Based on boundary condition (8), we have

$$
\begin{aligned}
B_{n} & =0, \\
A_{n} & =\frac{a^{n}}{n \pi C_{n}\left(a^{2 n}-b^{2 n}\right)}\left[\sin \left(n\left(\frac{\pi}{2}-\gamma\right)\right)-\sin \left(n\left(\frac{\pi}{2}+\gamma\right)\right)\right] \\
& =-\frac{2 a^{n} \cos (n \pi / 2) \sin n \gamma}{n \pi C_{n}\left(a^{2 n}-b^{2 n}\right)} .
\end{aligned}
$$

Thus,

$G(r, \phi)=-\frac{2}{\pi} \sum_{n=2,4,6}^{\infty} \frac{(a / b)^{n}(r / b)^{n}+(a / r)^{n}}{n\left[(a / b)^{2 n}-1\right]} \sin n \gamma \sin n\left(\phi+\frac{\pi}{2}\right)$.

Here, we suppose that $R=r / b$ and $\tau=a / b$, so $G$ can be written as

$$
G(r, \phi)=-\frac{2}{\pi} \sum_{n=2,4,6}^{\infty} \frac{R^{n}+R^{-n}}{n\left[\tau^{n}-\tau^{-n}\right]} \sin n \gamma \sin n\left(\phi+\frac{\pi}{2}\right) .
$$




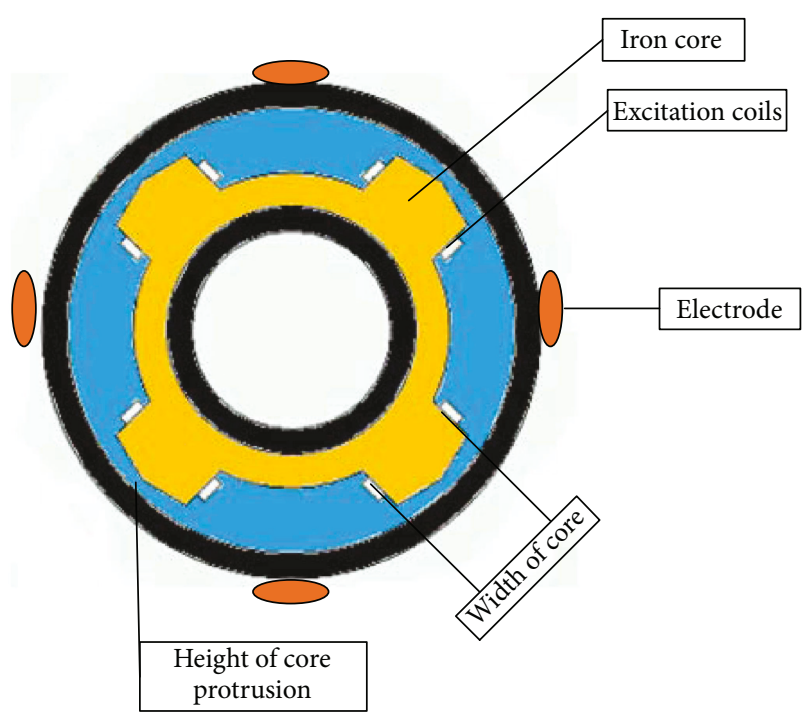

FIgURE 2: The schematic diagram of the excitation part of the annular flow electromagnetic measurement system.

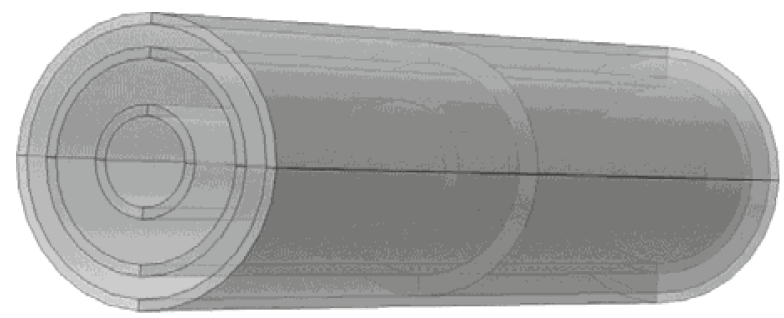

FIGURE 3: The 3D model of the annular four-electrode flow electromagnetic measurement system and the flow path.

The density of the virtual current can be derived using the gradient method based on (11). Theoretically, the shape and the parameters of the exciting coil can be solved based on the constant condition of the weight function vector. However, it is very complicated and difficult to derive the shape and the parameters of the exciting coil by the analytical method. Even if the result is solved, it is also complicated and difficult for the coil to wind. In recent years, numerical simulation has become an important process in a new system design. These difficulties can be worked out by the finite element software for multifields.

\section{Optimization of the Annular Four-Electrode Electromagnetic Flowmeter}

3.1. Optimization of Excitation Structure. To avoid winding the complicated coil, the iron core was introduced to the excitation part of the annular flow electromagnetic measurement system. Considering the special downhole environment, the schematic diagram of the excitation part of annular flow electromagnetic measurement system is shown in Figure 2. Here, the inner radius of the system is $4 \mathrm{~cm}$, the outer radius is $9 \mathrm{~cm}$, and the inner and outer wall thickness of the system is $1 \mathrm{~cm}$. In the annular flow electromagnetic measurement system, four excitation coils were positioned to generate a magnetic field. Four pick-up electrodes were located around the diameter of the external system wall, so that the flow-induced voltage could be detected. The 3D model of the annular four-electrode flow electromagnetic measurement system and the flow path by COMSOL are shown in Figure 3.

In this paper, the structure of the iron core and the size of the coil among the annular domain between the inner and outer surfaces of the system were investigated, and an optimized excitation structure was designed. Therefore, the width of the core and the height of core protrusion could be used as variables. In the simulations, the round electrodes were chosen with a radius of $0.7 \mathrm{~cm}$, and the length of the iron core was $10 \mathrm{~cm}$. The excitation coils were modified with the width of the core changing from $2 \mathrm{~cm}$ to $5 \mathrm{~cm}$ and the height of core protrusion from $5.5 \mathrm{~cm}$ to $8.5 \mathrm{~cm}$. The width of the core was incremented every $1 \mathrm{~cm}$, while the height of core protrusion every $1 \mathrm{~cm}$.

To validate the effectiveness of the optimization, some performances of the selected optimum annular flow electromagnetic measurement system were compared. Compared simulation results of the magnetic flux vector and weight function vector are shown from Figures 4-7.

Figures 4-7 show that the distribution of the magnetic flux vector and weight function vector changes with the width of the core and height of core protrusion changing. Considering the important role of weight function vector in optimization, the weight function vector distributions from Figures 4-7 are analyzed closely. The analysis results show that the weight function vector gets the smallest distribution range (from -0.03 to 0.32 ) when core_X is equal to $5.5 \mathrm{~cm}$ and Core_w is equal to $2 \mathrm{~cm}$; the weight function vector gets the biggest distribution range (from -0.05 to 0.66 ) when core_X is equal to $8.5 \mathrm{~cm}$ and Core_w is equal to $5 \mathrm{~cm}$; the distribution range of the weight function vector increases while the width of the core and height of core protrusion increase steadily, and increasing the width of the coil is much more pronounced.

3.2. The Change of Electrode Radius. After the structure of the excitation system of the measurement system was optimized and in order to improve the accuracy of the system, some simulations about the radius of the electrodes were used to improve the system performance. The electrodes were modified, with the radius changing from $1 \mathrm{~cm}$ to $5 \mathrm{~cm}$, and the radius of the electrodes was incremented every $0.5 \mathrm{~cm}$. Compared simulation results of the virtual current vector and weight function vector are shown in Figures 8 and 9 .

Figures 8 and 9 show that the virtual current density and weight function vector changed while the electrode radius changed. Based on Figures 8 and 9, we can find that the weight function vector gets the smallest distribution range (from 0 to 0.03 ) when the electrode radius is $5 \mathrm{~cm}$; the weight function vector gets the biggest distribution range (from -0.01 to 0.16 ) when the electrode radius is $1 \mathrm{~cm}$; the distribution range of weight function vector decreases while the electrode radius increases steadily. 

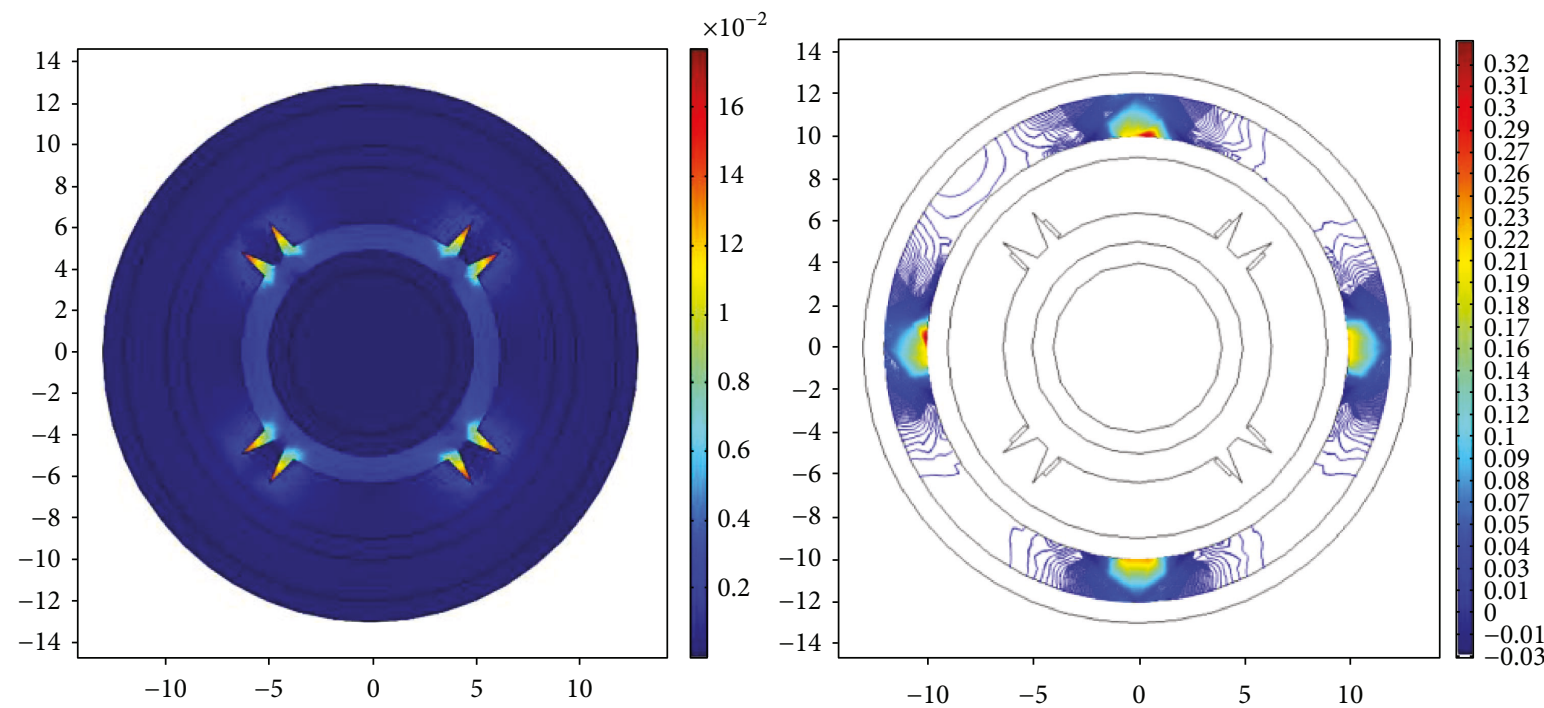

(a) When the height of core protrusion is $5.5 \mathrm{~cm}$
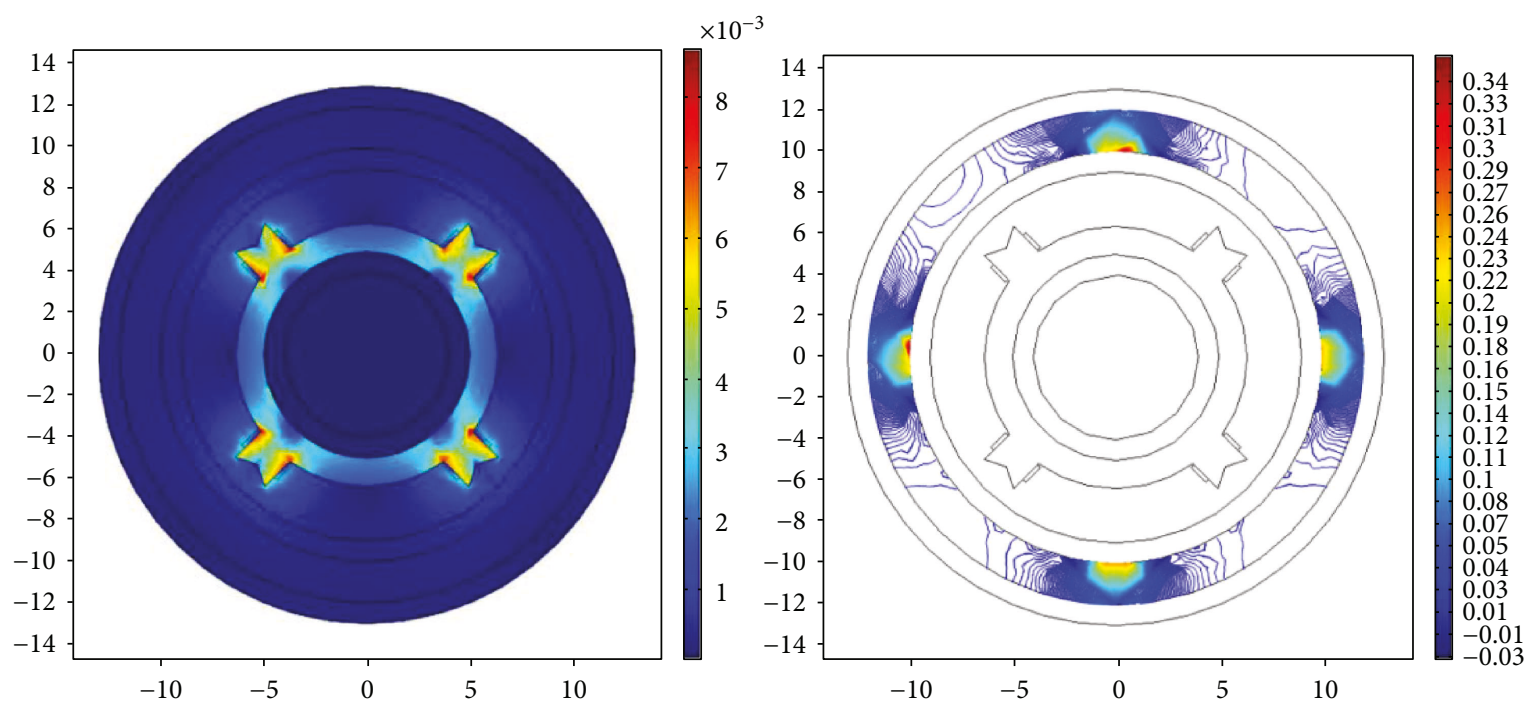

(b) When the height of core protrusion is $7.5 \mathrm{~cm}$

FIgURE 4: The simulation diagram of the magnetic flux vector and weight function vector with core width equal to $2 \mathrm{~cm}$.

\section{Results and Discussion}

\subsection{Evaluation Indexes}

4.1.1. Standard Deviation of the Weight Function Vector. The ideal excitation system design is to make the weight function vector to be a constant, and this is helpful to improve the measurement accuracy of the system. However, the weight function vector is not easily designed to be a constant. In order to design a magnetic field that makes the weight function vector as constant as possible, it is necessary to define a design quantity that measures the degree of nonuniformity of the weight function vector over the cross-sectional area of the annular domain. The definition of the design quantity was discussed by Dennis and Wyatt in 1972 [13], but owing to integration difficulties, this quantity is not used here. Instead, a similar quantity $\mathbf{W}_{\sigma}$ named the weight function vector standard deviation is used which is defined by the following:

$$
\mathbf{W}_{\sigma}=\sqrt{\frac{\sum_{i=1}^{N}\left(\mathbf{W}_{i}-\overline{\mathbf{W}}\right)^{2}}{N}},
$$

where $\overline{\mathbf{W}}$ is the average weight function vector value of all points in the $X Y$ plane of the annular while $z=0$ and $N$ is the number of the points.

4.1.2. Homogeneity Range Ratio of Weight Function Vector. To evaluate the homogeneity range in the annular area, the ratio of the homogeneity range is used as an evaluation criterion [14]. The equation of this evaluation criterion is as follows: 

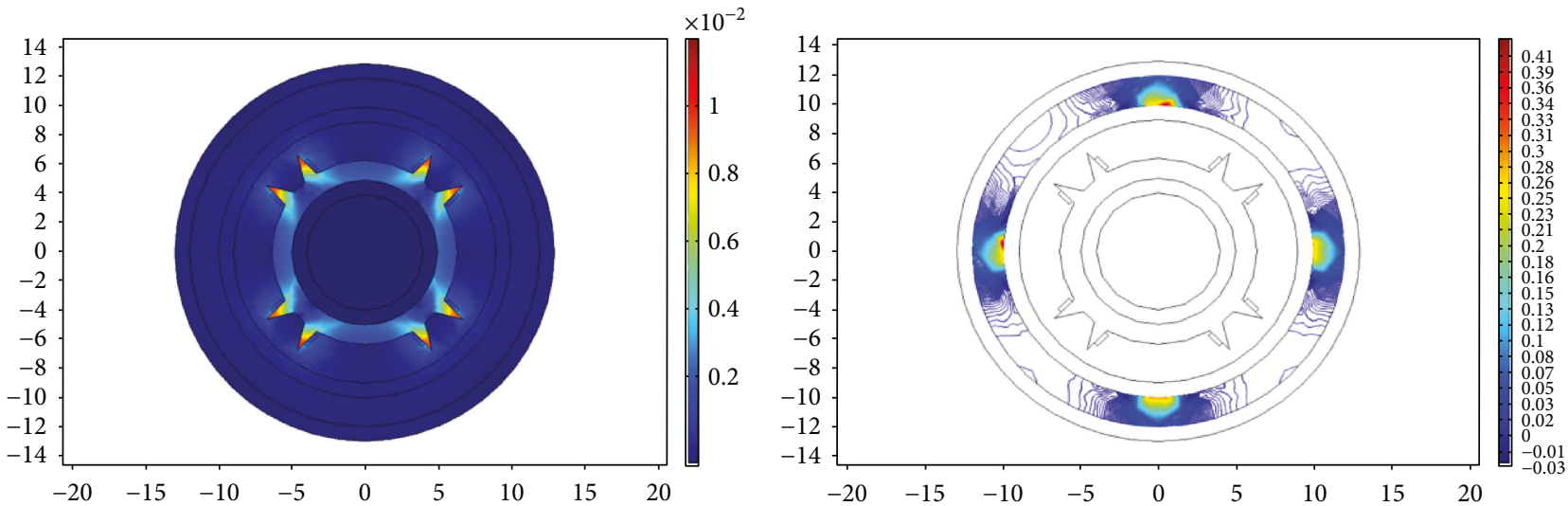

(a) When the height of core protrusion is $5.5 \mathrm{~cm}$
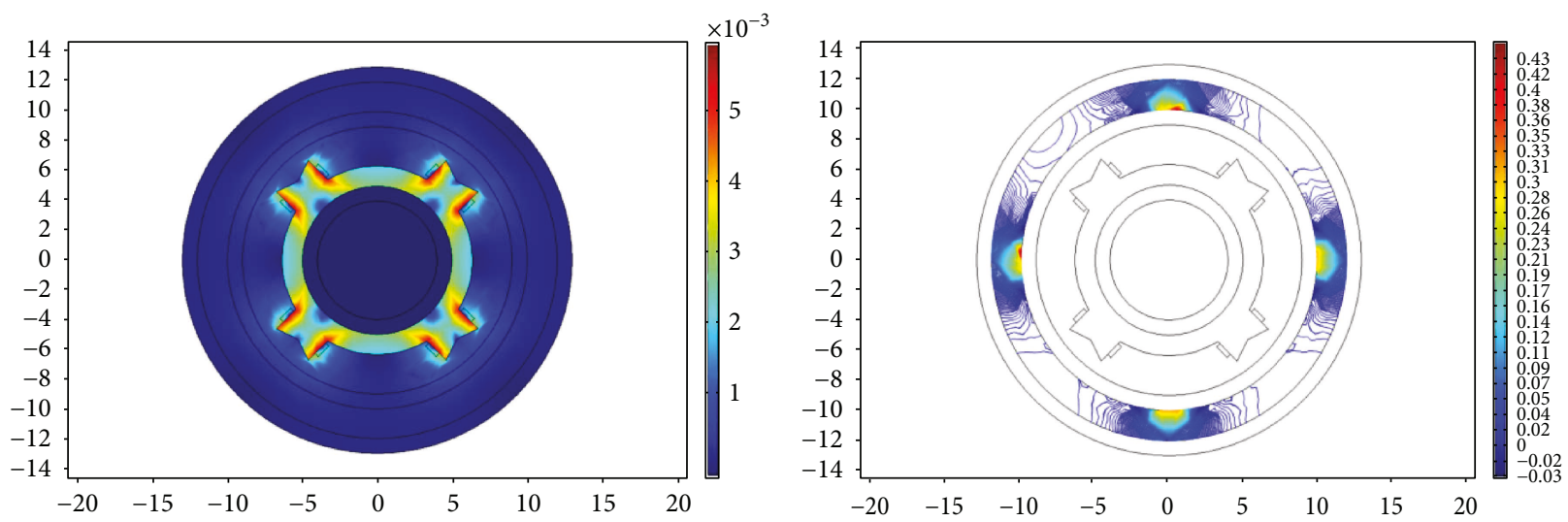

(b) When the height of core protrusion is $7.5 \mathrm{~cm}$

FIgURE 5: The simulation diagram of the magnetic flux vector and weight function vector with core width equal to $3 \mathrm{~cm}$.

$$
\frac{\left|\mathbf{W}_{(x, y)}-\overline{\mathbf{W}}\right|}{\overline{\mathbf{W}}} \times 100 \% \leq P
$$

where $\mathbf{W}_{(x, y)}$ is the weight function vector of the central cross section of the electrode in the annular area, $\overline{\mathbf{W}}$ is the average weight function vector value of all points in the $X Y$ plane of the annular while $z=0$, and $P$ is a uniform percentage value that depends on actual distribution and which is $30 \%$ in this research.

If a finite element meets (13), we may regard the area of this finite element to be homogeneous; otherwise, we regard that the area of this finite element is inhomogeneous. If the number of inhomogeneous finite elements is $N_{1}$ and the number of homogeneous finite elements is $N_{2}$, the homogeneity range ratio can be defined as follows:

$$
P_{e}=\frac{N_{2}}{N_{1}+N_{2}} \times 100 \% .
$$

4.1.3. The Coefficient of Variation of Weight Function Vector. The coefficient of variation is a useful statistic for comparing the degree of variation from one data series to another, even if the means are drastically different from one another. A coefficient of variation of the weight function vector is a statistical measure of the dispersion of the weight function vector data points in a data series around the mean. It is calculated as follows:

$$
C_{v}=\frac{\mathbf{W}_{\sigma}}{\overline{\mathbf{W}}},
$$

where $\mathbf{W}_{\sigma}$ is the standard deviation of the weight function vector and $\overline{\mathbf{W}}$ is the average weight function vector value of all points.

4.1.4. The Sensitivity of Output Voltage. The fourth performance criterion is the sensitivity of the output voltage which gives the strength of the system output. In this research, we assure that the turns and current of the coil are constant, and we get the sensitivity of the output voltage while the average flow velocity is 1 meter per second.

During the optimal design and quantitative evaluation process, smaller standard deviation of the weight function vector is better, as is a bigger homogeneity range ratio of the weight function vector is better, smaller coefficient of variation of the weight function vector is better, and bigger sensitivity of the output voltage is better. In these four evaluation indexes, the standard deviation of the vector weight function is the most important evaluation index, and the other 3 indexes are auxiliary reference indexes. 

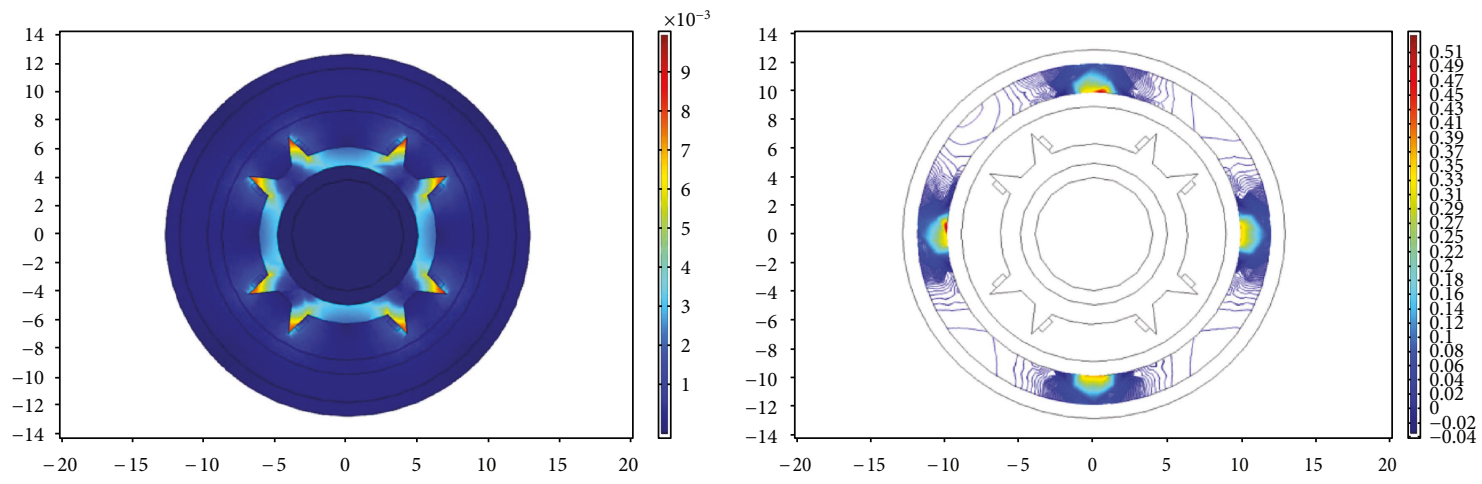

(a) When the height of core protrusion is $5.5 \mathrm{~cm}$
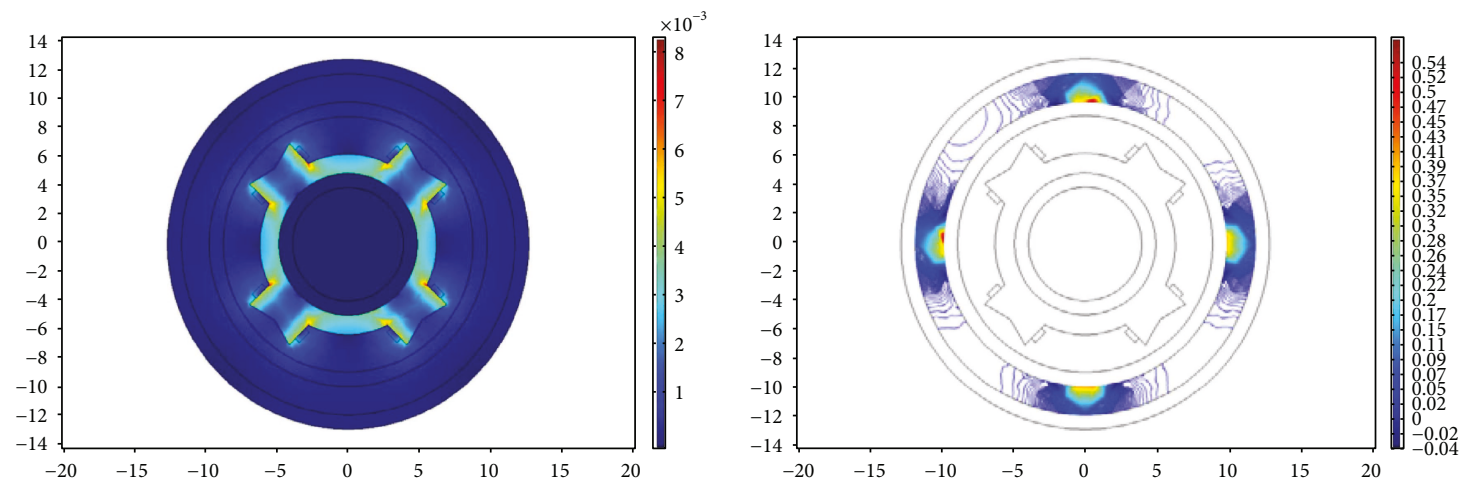

(b) When the height of core protrusion is $7.5 \mathrm{~cm}$

FIgURE 6: The simulation diagram of the magnetic flux vector and weight function vector with core width equal to $4 \mathrm{~cm}$.
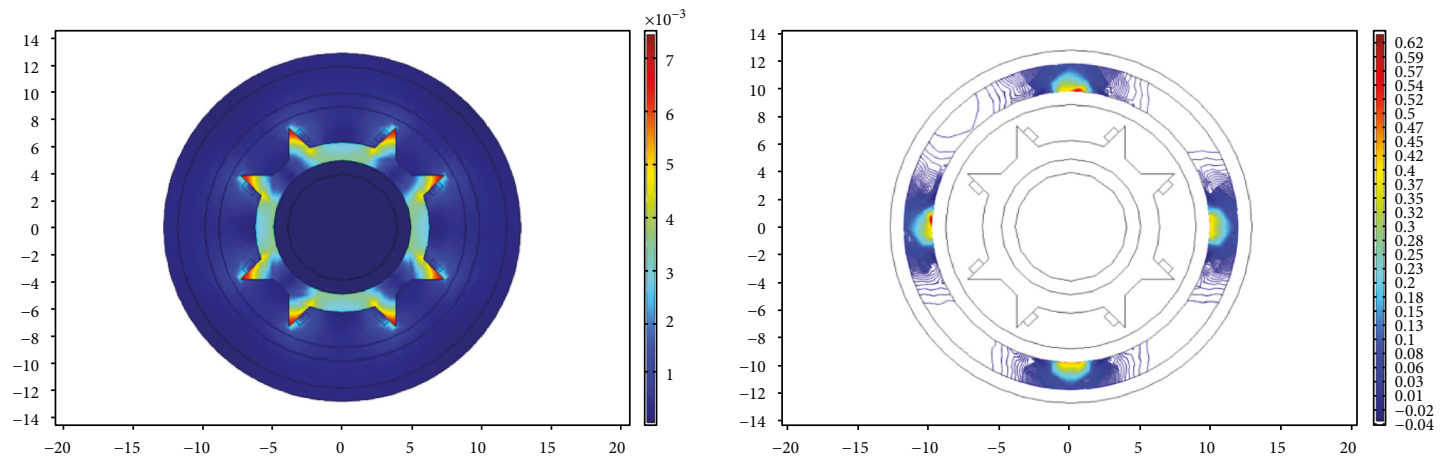

(a) When the height of core protrusion is $5.5 \mathrm{~cm}$
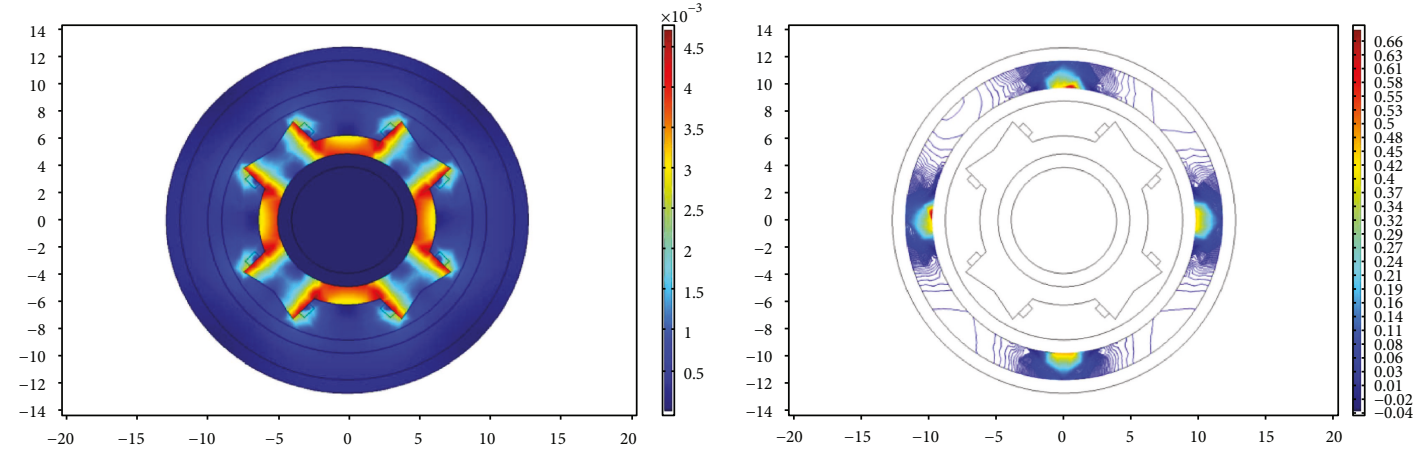

(b) When the height of core protrusion is $7.5 \mathrm{~cm}$

FIgURE 7: The simulation diagram of the magnetic flux vector and weight function vector with core width equal to $5 \mathrm{~cm}$. 


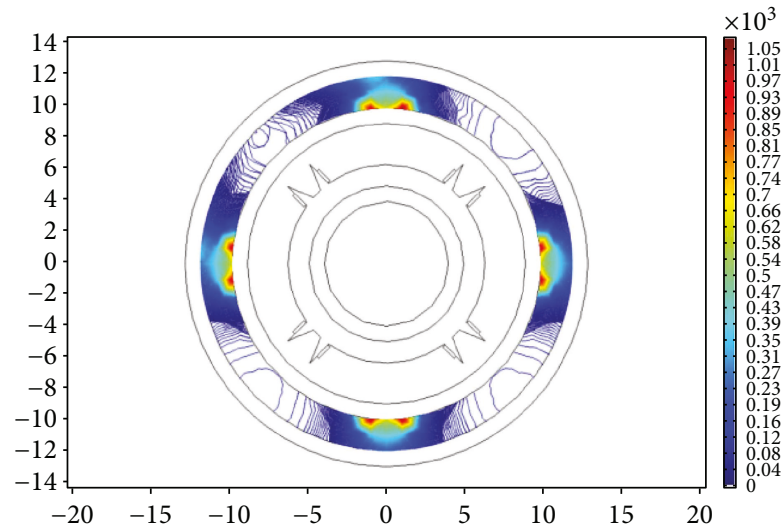

(a) Radius equal to $1 \mathrm{~cm}$ in the $X Y$ plane

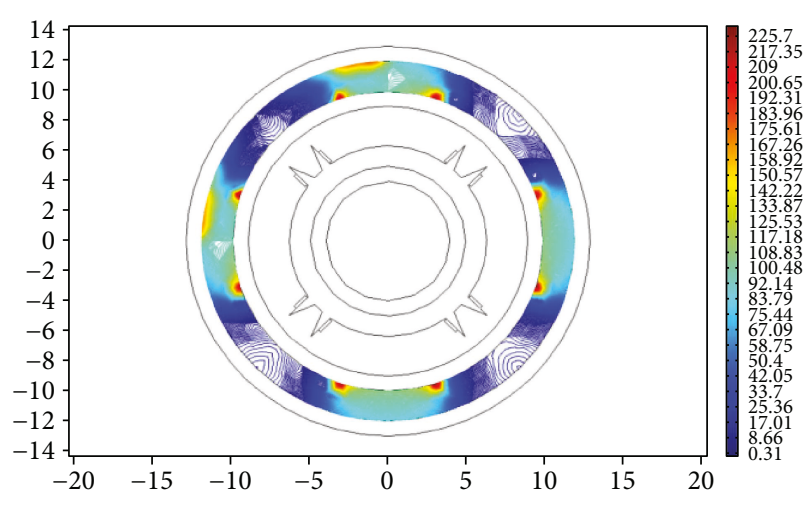

(c) Radius equal to $3 \mathrm{~cm}$ in the $X Y$ plane

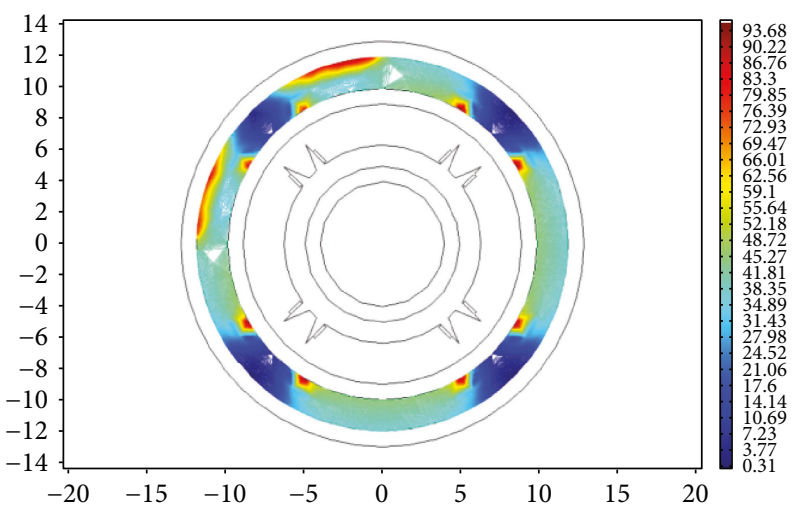

(e) Radius equal to $5 \mathrm{~cm}$ in the $X Y$ plane

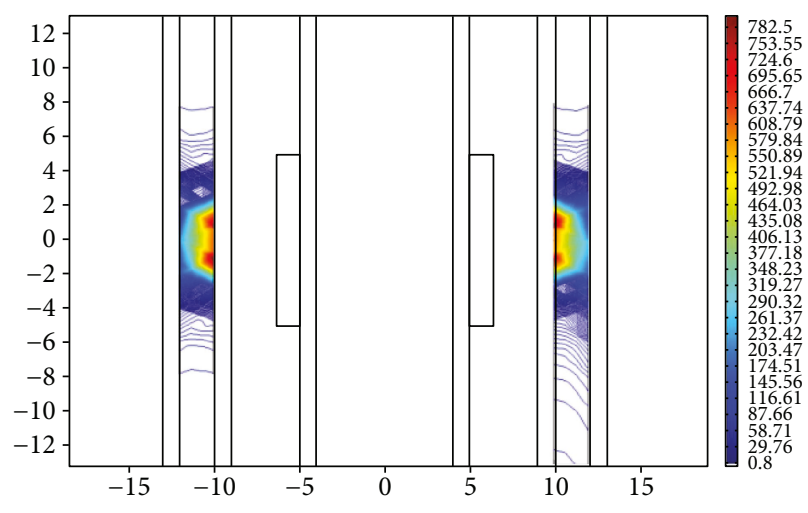

(b) Radius equal to $1 \mathrm{~cm}$ in the $Y Z$ plane

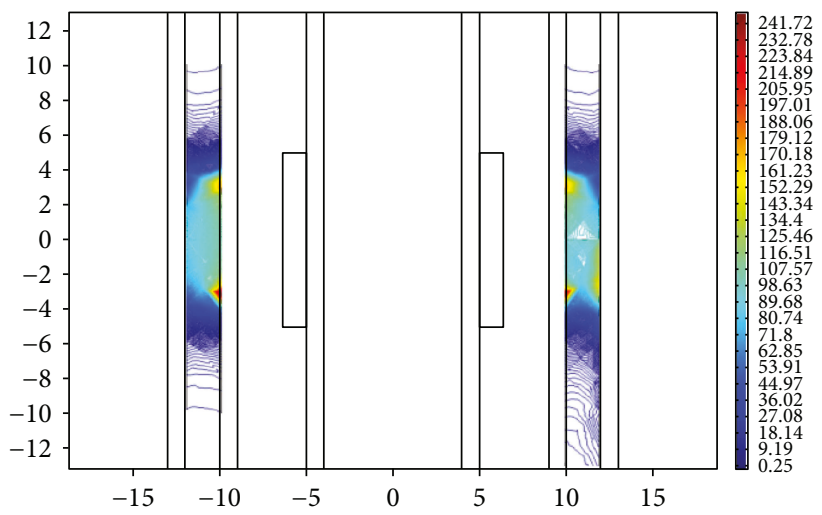

(d) Radius equal to $3 \mathrm{~cm}$ in the $Y Z$ plane

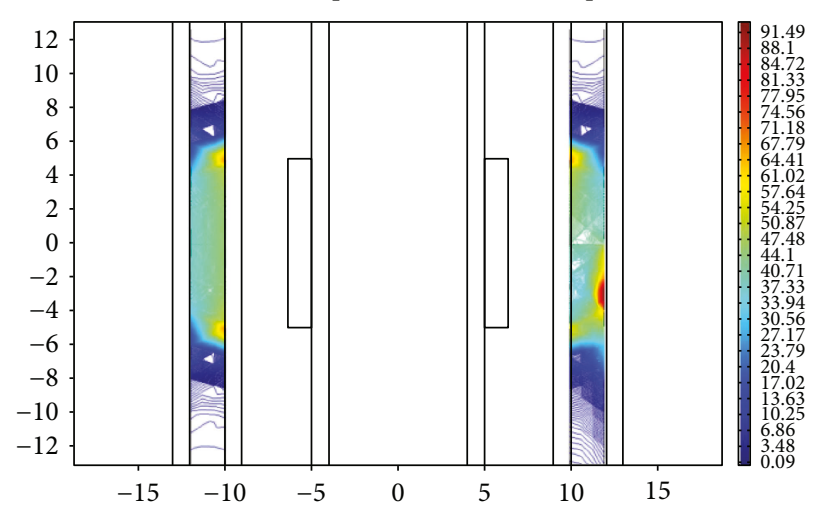

(f) Radius equal to $5 \mathrm{~cm}$ in the $Y Z$ plane

Figure 8: The simulation diagram of virtual current density with electrode radius equal to $1 \mathrm{~cm}, 3 \mathrm{~cm}$, and $5 \mathrm{~cm}$ separately.

4.2. Analysis of the Excitation System Optimization Effect. In order to intuitively analyze and compare the effect of excitation structure optimization, the value of the weight function vector standard deviation is analyzed by the definition of the design quantity. To validate the effectiveness of the optimization, the results of the weight function vector standard deviation are compared in Figure 10.

In Figure 10, Core_w represents the width of the core and core_X represents the height of core protrusion. When the width of the core is fixed, the weight function vector standard deviation increases while the height of core protrusion increases steadily. When the height of core protrusion is fixed, the weight function vector standard deviation increases while the position of the width of the core increases steadily.

Using Figure 10, we can find the minimum standard deviation of the weight function vector while core_X is $5.5 \mathrm{~cm}$ and Core_w is $2 \mathrm{~cm}$. Similarly, based on the simulation data and mathematical operations, the variation curve of the homogeneity range ratio, coefficient of variation of the weight function vector, and the sensitivity of the output voltage are shown from Figures 11-13.

It can be seen from Figures 11-13 that when the width of the core and height of core protrusion change, the evaluation indexes change. When core_X is equal to $5.5 \mathrm{~cm}$ and Core_w 


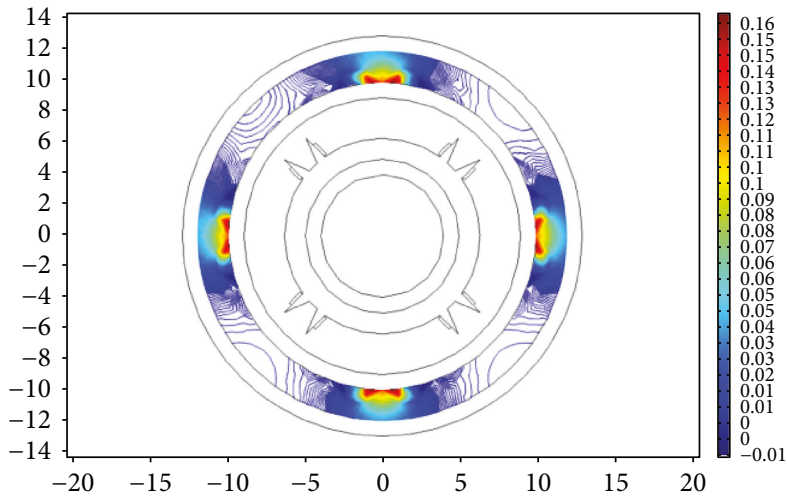

(a) Radius equal to $1 \mathrm{~cm}$ in the $X Y$ plane

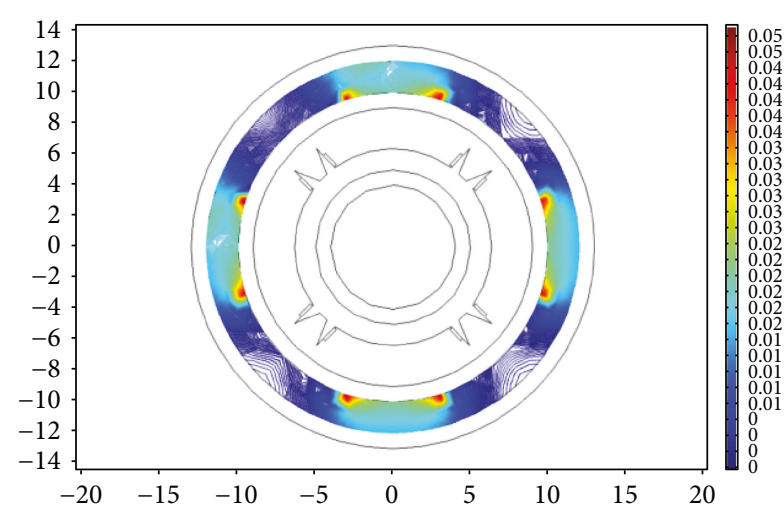

(c) Radius equal to $3 \mathrm{~cm}$ in the $X Y$ plane

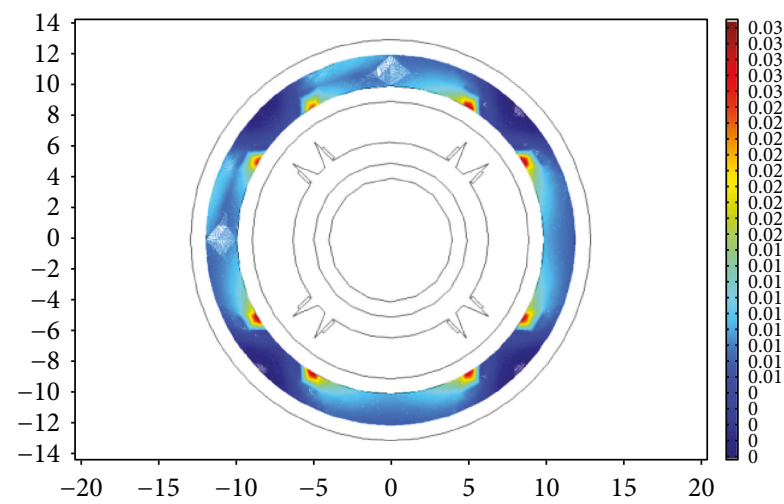

(e) Radius equal to $5 \mathrm{~cm}$ in the $X Y$ plane

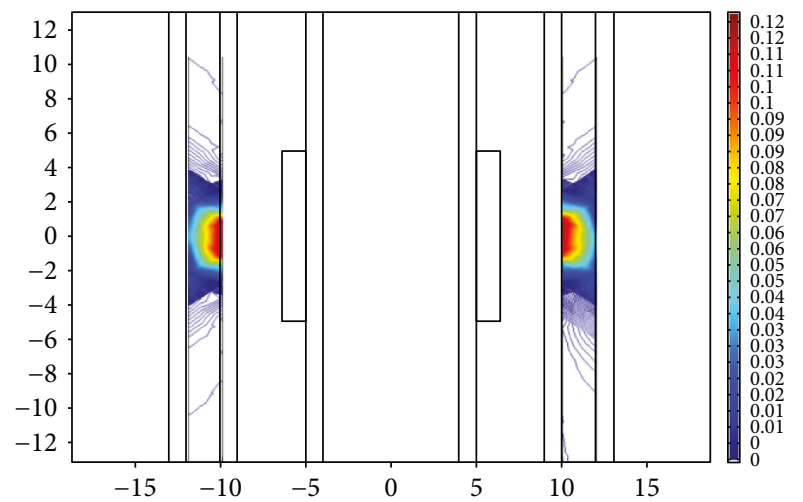

(b) Radius equal to $1 \mathrm{~cm}$ in the $Y Z$ plane

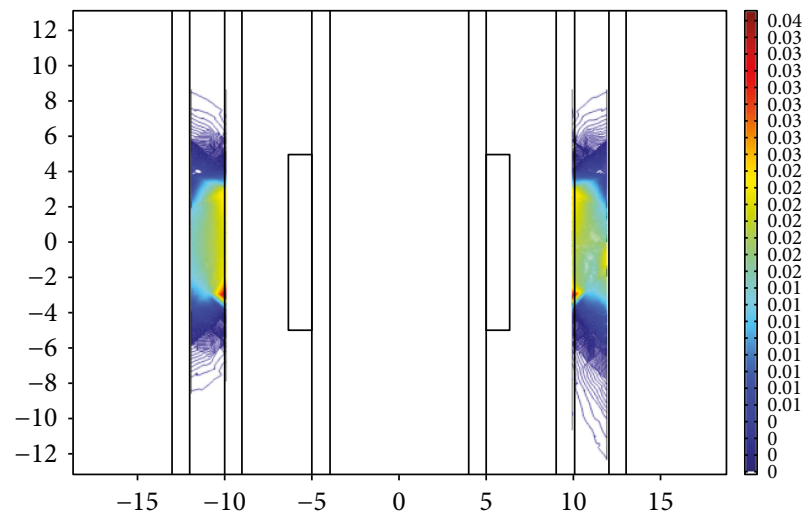

(d) Radius equal to $3 \mathrm{~cm}$ in the $Y Z$ plane

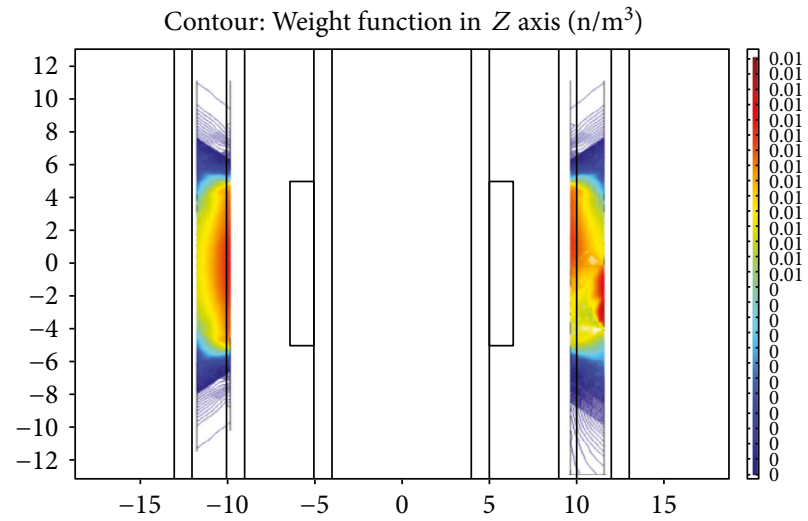

(f) Radius equal to $5 \mathrm{~cm}$ in the $Y Z$ plane

Figure 9: The simulation diagram of weight function vector with electrode radius equal to $1 \mathrm{~cm}, 3 \mathrm{~cm}$, and $5 \mathrm{~cm}$ separately.

is equal to $2 \mathrm{~cm}$, the homogeneity range ratio is relatively large, but the coefficient of variation of the weight function is large and the sensitivity of output voltage is small.

Considering that the standard deviation of the vector weight function is the most important evaluation index, optimum results of annular flow electromagnetic measurement system under the double pairs of coil excitation structure and current finite structure space can be obtained, with core_X $=5.5 \mathrm{~cm}$ and Core_w $=2 \mathrm{~cm}$ being the best coil excitation structures. However, from the range of numerical magnitude of evaluation indexes, we can find that changing the width of the core and height of core protrusion has a limited effect on improving the system measurement performance.

4.3. Analysis of Electrode Radius Effect. In order to intuitively analyze and compare the effect of excitation system optimization, the value of the weight function vector standard deviation can be analyzed using the definition of the design quantity. To validate the effectiveness of the optimization, the results of the weight function vector standard deviation are compared in Figure 14. The data result in Figure 14 shows that the weight function vector standard deviation decreases while the electrode radius increases steadily, so the big radius 


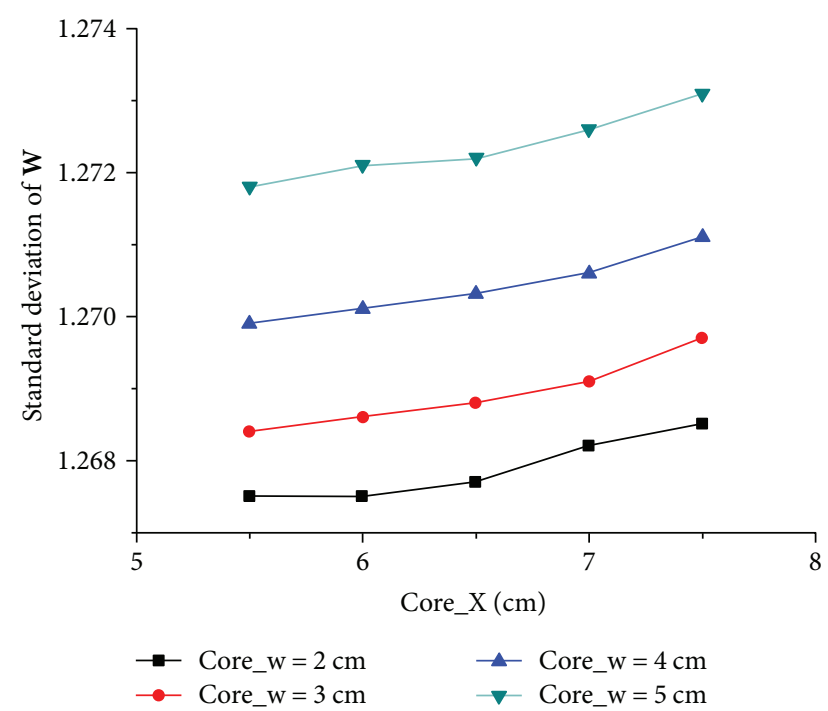

FIGURE 10: The curve of weight function vector standard deviation and structure of excitation system.

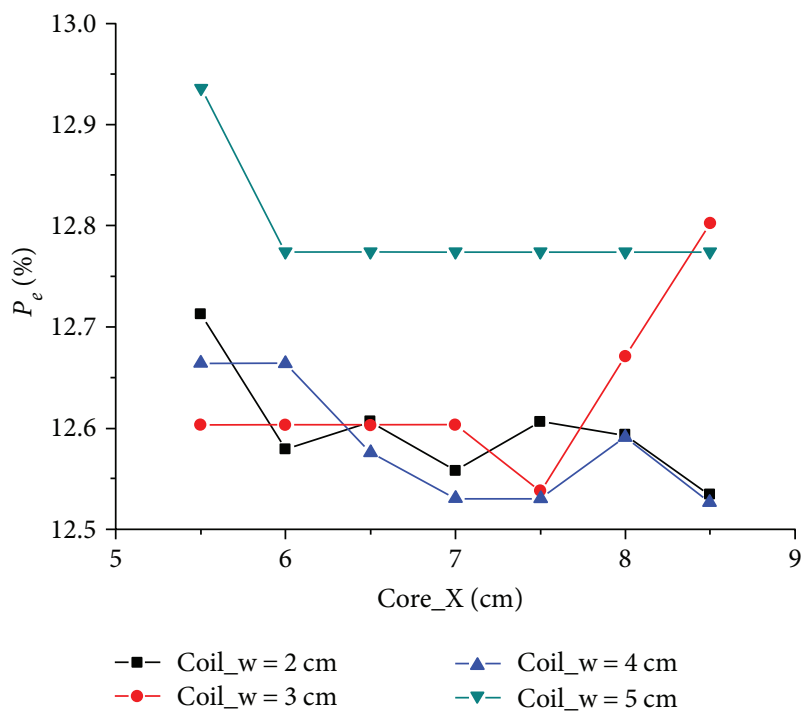

Figure 11: The curve of the homogeneity range ratio and structure of the excitation system.

electrode enhances the measured precision of annular flow electromagnetic measurement system.

Similarly, based on simulation data and mathematical operations, the variation curve of the homogeneity range ratio, the coefficient of variation of the weight function vector, and the sensitivity of output voltage are shown from Figures 15-17. It is clear that with the increases of the electrode radius from $0.5 \mathrm{~cm}$ to $6 \mathrm{~cm}$, the homogeneity range ratio keeps increasing from $6.43 \%$ to $79.49 \%$, the coefficient of variation of the weight function vector keeps decreasing from 2410 to 498 , and the sensitivity of the output voltage increases first from $3.07 E-05 \mathrm{~V}$ to $3.22 E-05 \mathrm{~V}$ and then decreases from $3.22 E-05 \mathrm{~V}$ to $2.81 E-05 \mathrm{~V}$. Thus, the electrode radius need to be chosen according to the requirement

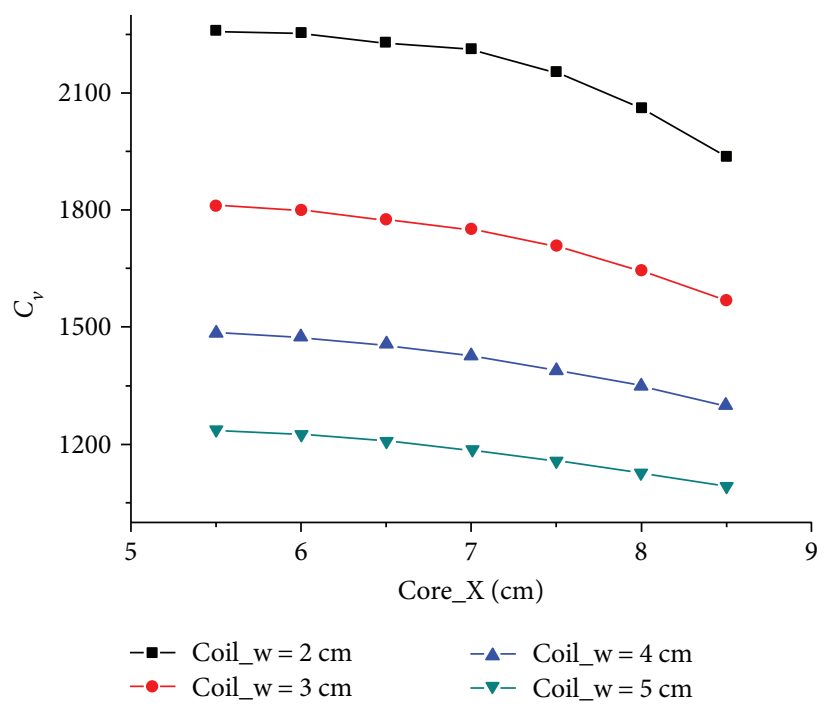

FIgURE 12: The curve of the coefficient of variation of weight function and structure of the excitation system.

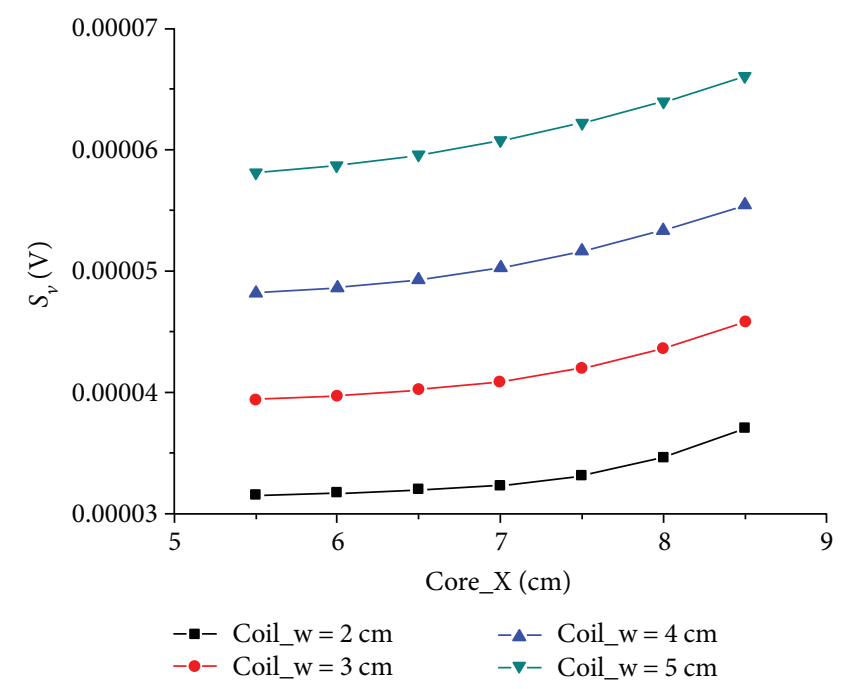

FIGURE 13: The curve of the sensitivity of the output voltage and the structure of excitation system.

of the system performance, and the electrode radius of $4.5 \mathrm{~cm}$ has a better optimum comprehensive effect during the annular flow measuring process.

\section{Conclusions}

This paper described the design and optimization of annular flow electromagnetic measurement system for drilling engineering. The following conclusions can be drawn according to the above-mentioned analysis:

(1) The theory of annular flow electromagnetic measuring and optimization principles were described, and an annular flow electromagnetic measurement system simulation model was built based on this theory by COMSOL. 


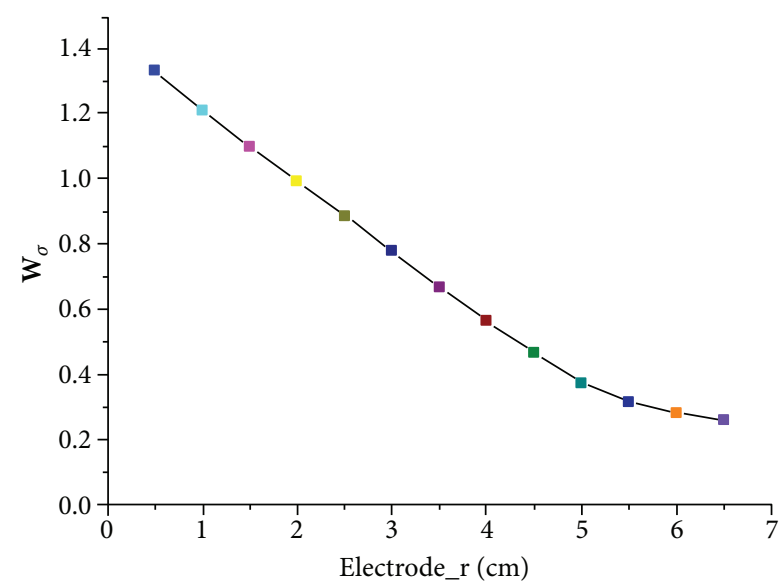

FIGURE 14: The curve of the weight function vector and electrode radius.

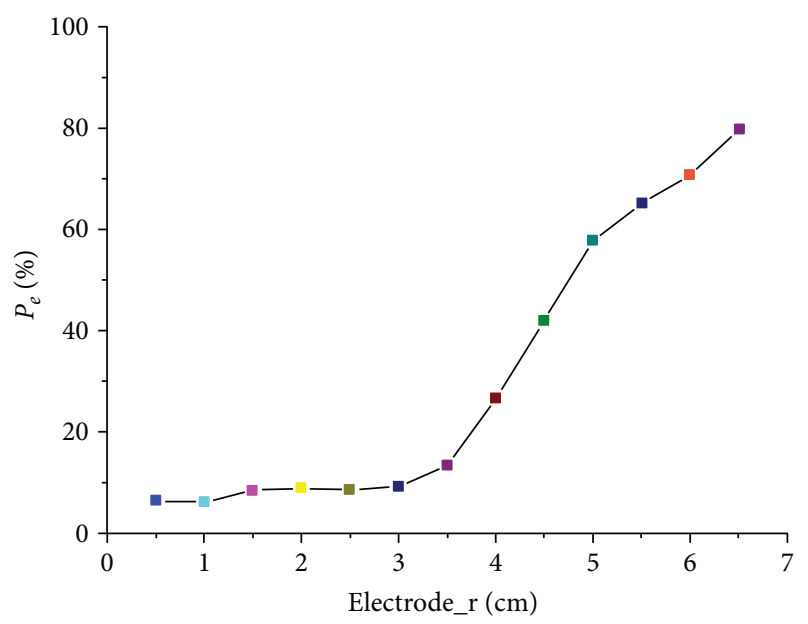

FIGURE 15: The curve of the homogeneity range ratio and electrode radius.

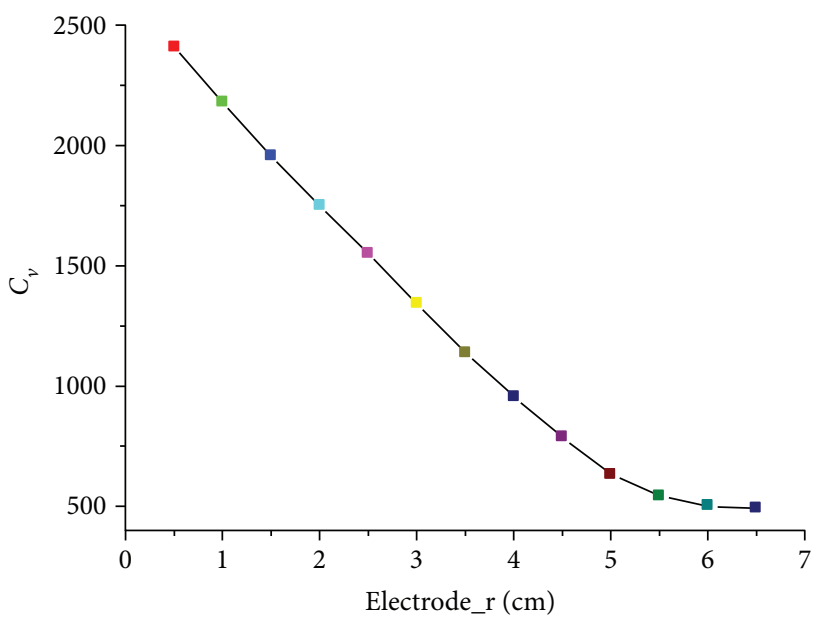

Figure 16: The curve of the coefficient of variation of weight function and electrode radius.

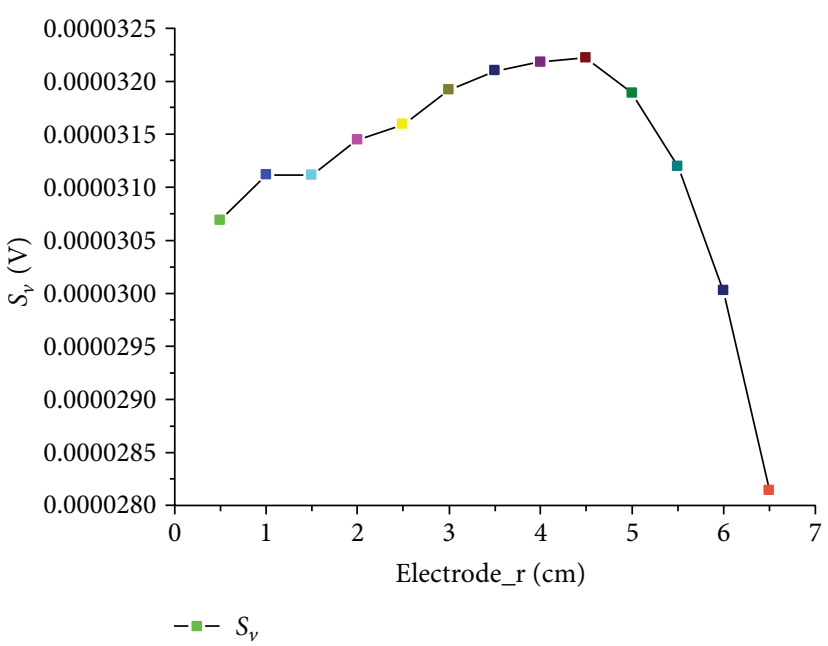

FIgURE 17: The curve of the sensitivity of the output voltage and electrode radius.

(2) Simulations on the structure of excitation system of measurement system were carried out, as well as some simulations on the size of the electrode's radius were also carried based on the optimized structure, and then all the simulation results were analyzed to evaluate the optimization effects based on the evaluation indexes. The simulation results showed that the optimized structure of excitation system and electrode radius size can yield a better performance during the annular flow measurement process.

\section{Conflicts of Interest}

The authors declare that there is no conflict of interest regarding the publication of this article.

\section{Acknowledgments}

This work is supported by Open Fund (OGE201702-19) of Key Laboratory of Oil \& Gas Equipment of Ministry of Education (Southwest Petroleum University), the National Natural Science Foundation (no. 51504211), and the State Administration of National Security (no. sichuan-00112016AQ).

\section{References}

[1] A. Aqeel and M. S. Butt, "The relationship between energy consumption and economic growth in Pakistan," Asia-Pacific Development Journal, vol. 8, no. 2, 2001.

[2] L. Ge, G. Wei, Q. Wang, Z. Hu, and J. Li, "Novel annular flow electromagnetic measurement system for drilling engineering," IEEE Sensors Journal, vol. 17, no. 18, pp. 5831-5839, 2017.

[3] M. K. Bevir, "The theory of induced voltage electromagnetic flowmeters," Journal of Fluid Mechanics, vol. 43, no. 3, pp. 577-590, 1970.

[4] B. Horner, F. Mesch, and A. Trachtler, "A multi-sensor induction flowmeter reducing errors due to non-axisymmetric flow 
profiles," Measurement Science and Technology, vol. 7, no. 3, pp. 354-360, 1996.

[5] B. Horner, "A novel profile-insensitive multi-electrode induction flowmeter suitable for industrial use," Measurement, vol. 24, no. 3, pp. 131-137, 1998.

[6] A. Michalski, J. Starzynski, and S. Wincenciak, "Optimal design of the coils of an electromagnetic flow meter," IEEE Transactions on Magnetics, vol. 34, no. 5, pp. 2563-2566, 1998.

[7] J. Z. Wang, G. Y. Tian, and G. P. Lucas, "Relationship between velocity profile and distribution of induced potential for an electromagnetic flow meter," Flow Measurement and Instrumentation, vol. 18, no. 2, pp. 99-105, 2007.

[8] A. Michalski, J. Starzynski, and S. Wincenciak, "Electromagnetic flowmeters for open channels - two-dimensional approach to design procedures," IEEE Sensors Journal, vol. 1, no. 1, pp. 52-61, 2001.

[9] D. A. G. Vieira, A. C. Lisboa, and R. R. Saldanha, "An enhanced ellipsoid method for electromagnetic devices optimization and design," IEEE Transactions on Magnetics, vol. 46, no. 8, pp. 2843-2851, 2010.

[10] K. W. Lim and M. K. Chung, "Relative errors in evaluating the electromagnetic flowmeter signal using the weight function method and the finite volume method," Flow Measurement and Instrumentation, vol. 9, no. 4, pp. 229-235, 1998.

[11] A. Michalski, J. Starzynski, and S. Wincenciak, "3-D approach to designing the excitation coil of an electromagnetic flowmeter," IEEE Transactions on Instrumentation and Measurement, vol. 51, no. 4, pp. 833-839, 2002.

[12] W. Yin, A. J. Peyton, F. Stefani, and G. Gerbeth, "Theoretical and numerical approaches to the forward problem and sensitivity calculation of a novel contactless inductive flow tomography (CIFT)," Measurement Science and Technology, vol. 20, no. 10, article 105503, 2009.

[13] J. Dennis and D. G. Wyatt, "Effect of hematocrit value upon electromagnetic flowmeter sensitivity," Circulation Research, vol. 24, no. 6, pp. 875-886, 1969.

[14] O. Cazarez, D. Montoya, A. G. Vital, and A. C. Bannwart, "Modeling of three-phase heavy oil-water-gas bubbly flow in upward vertical pipes," International Journal of Multiphase Flow, vol. 36, no. 6, pp. 439-448, 2010. 


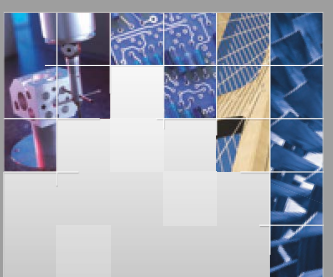

\section{Enfincering}
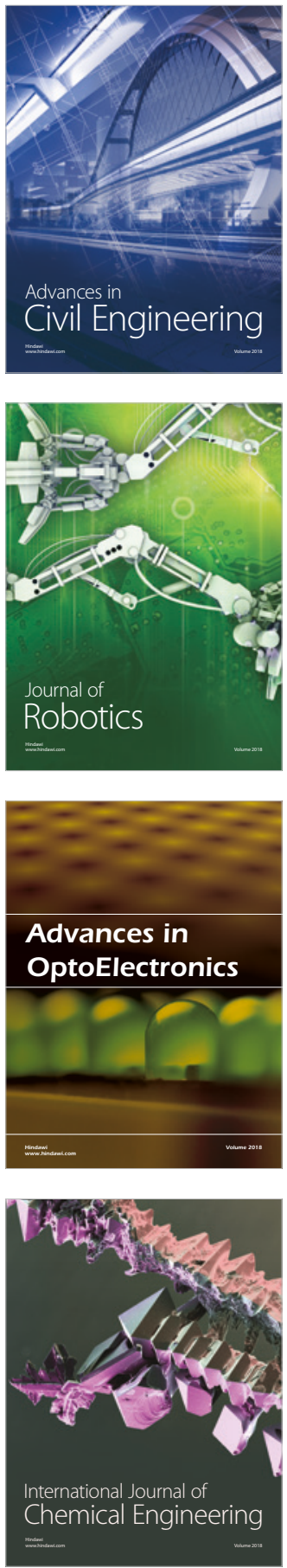

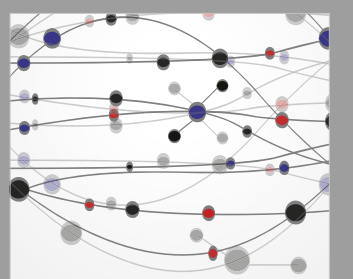

\section{Rotating \\ Machinery}

The Scientific World Journal

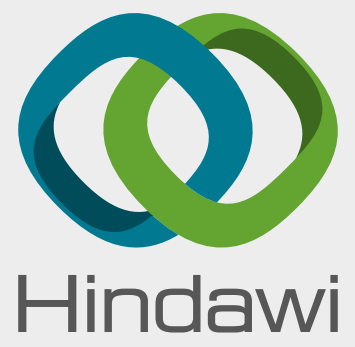

Submit your manuscripts at

www.hindawi.com
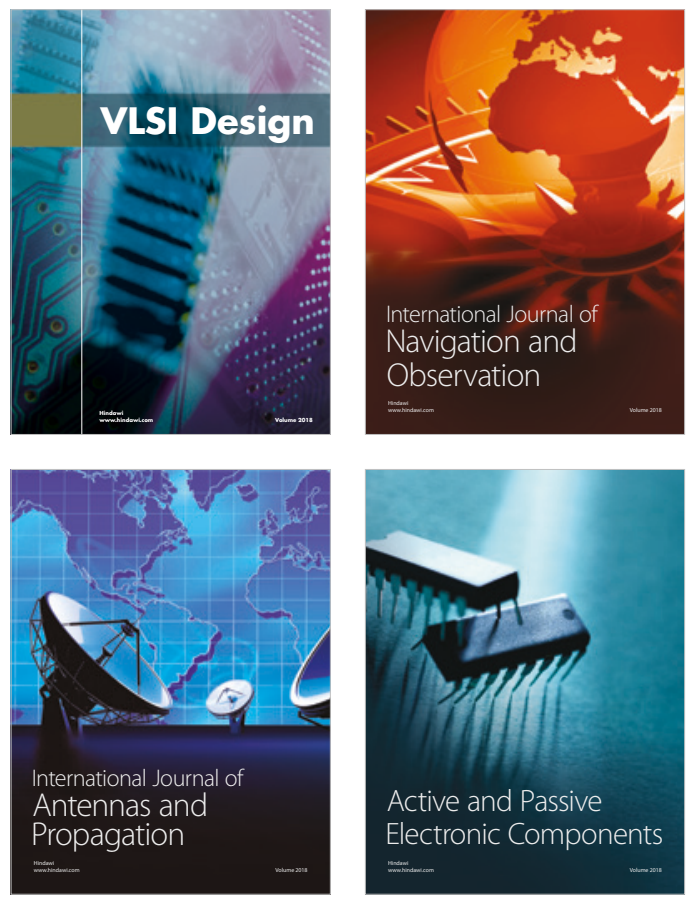
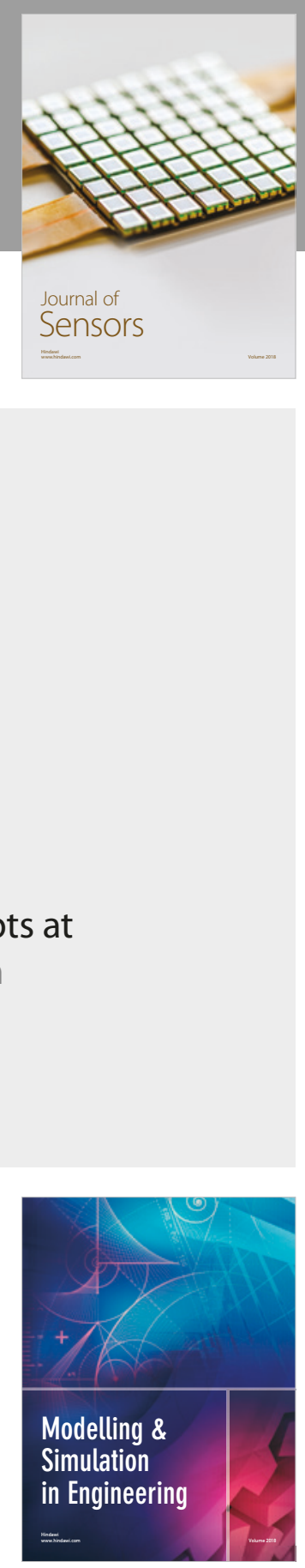

\section{Advances \\ Multimedia}
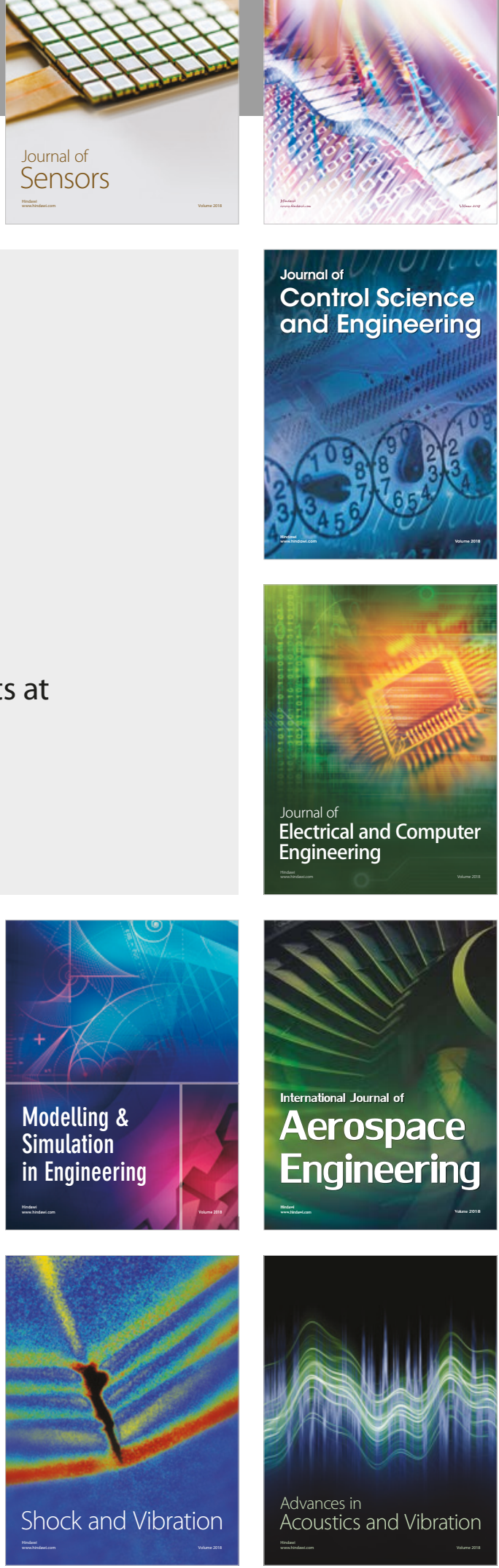